\title{
Designing the User Experience for C4ISR Systems in the U.S. Army ${ }^{*}$
}

\author{
Pamela Savage-Knepshield, Jeffrey Thomas, Christopher Paulillo, \\ James Davis, Diane Quarles, and Diane Mitchell \\ U.S. Army Research Laboratory, Human Research and Engineering \\ Directorate, Aberdeen Proving Ground, MD, USA \\ \{pamela.a.savage-knepshield, jeffrey.a.thomas132, \\ christopher.r.paulillo, james.a.davis531, diane.1.quarles, \\ diane.k.mitchell\}.civ@mail.mil
}

\begin{abstract}
A unique set of challenges exist for implementing user-centered design principles in the context of military acquisition over and above those typically encountered by user experience designers. This paper focuses on the tools and techniques that we have utilized to help ensure that a positive user experience (UX) will result when Soldiers and systems interact under harsh conditions on the battlefield. Insights gained from applying these techniques to system design and evaluation early in the acquisition process and the impact that their use has had on training and system design are discussed.
\end{abstract}

Keywords: Agile development incorporating DUXU, design philosophy and DUXU, usability methods and tools.

\section{The U.S. Army MANPRINT Program's Focus on UX}

MANPRINT or Manpower and Personnel Integration was incorporated in the U.S. Army during the mid-1980's and is the U.S. Army's implementation of human systems integration. It forced a radical change in the way that defense contractors did business with the U.S. Army, requiring them to focus on the human and on the design of systems that would better fit Soldiers' needs and capabilities [1]. This is significant because in the military stakes are high; failures in human system interaction at the user interface (UI) not only result in loss of productivity, but also contribute to catastrophic incidents with loss of life and limb. Military system acquisition design successes as well as failures across the joint services have been documented in the public press and scientific and technical publications. And, in concert with efforts by organizations such as the National Research Council, whose mission is to improve government decision making and public policy, have raised awareness of the importance of considering human factors and human systems integration earlier in the acquisition process and have positively impacted acquisition policy and procedures [2].

The U.S. Army's MANPRINT program places the user - the American Soldier squarely in the center of the design process to ensure that their needs are the foremost

\footnotetext{
${ }^{*}$ The rights of this work are transferred to the extent transferable according to title 17 U.S.C. 105.
} 
consideration when making design trade-offs and decisions [3]. The processes and procedures used to procure equipment have evolved to meet the urgent demands of our forces in the field. Most notably is the U.S. Army's Agile Process, which has sought to keep pace with technological advances and accelerate the pace of network modernization [4]. The Agile process seeks to meet capability gaps by rapidly integrating emerging Command, Control Communications, Computers, Intelligence, Surveillance and Reconnaissance (C4ISR) systems and iteratively testing their effectiveness during Network Integration Evaluation (NIE) test events. This process has raised further challenges for UX designers as we seek to understand our users' goals, intentions, and behaviors and use this information to shape the behavior of the technology so Soldier-system interaction appears natural to the user. Lessons learned including what has worked well and what has not as we move to become more fully engaged earlier in the Agile and "traditional" acquisition processes are presented.

\section{Cognitive Workload Modeling and Simulation of the UX}

Modeling \& Simulation (M\&S) tools developed by the U.S. Army to support MANPRINT practitioners help ensure that the UX is optimized throughout the acquisition cycle. One of the key principles of UX is to incorporate user feedback as a design evolves. MANPRINT practitioners do this by incorporating user feedback as inputs to the models they develop during each phase of the acquisition cycle. They develop their models early in the cycle during the Analysis of Alternatives phase [5], the technology demonstration phase $[6,7]$, and continue throughout the acquisition cycle [8].

Using the U.S. Army-developed tool, the Improved Performance Research Integration Tool (IMPRINT), to design the UX for a C4ISR system, analysts adhere to the experimental design process replacing experiments with models in the process. They conduct cognitive task interviews with Soldier subject matter experts (SSMEs) and include the data obtained as a key step in their model development process. This step is critical because each IMPRINT model is a hierarchical network of the mission, functions, and tasks Soldiers perform with the interface associated with the C4ISR system. Tasks within the models are verified by the SSMEs and modified as necessary to ensure user feedback is a part of the M\&S process. Each model run is a dynamic, stochastic simulation of Soldiers performing their tasks with the C4ISR system. Once verified, the models are run multiple times.

Multiple IMPRINT models can represent C4ISR systems with alternative designs. By comparing mission performance across the models, analysts can predict the impact of the alternative C4ISR UI designs on mission performance. The output IMPRINT provides to analysts for comparisons can include mission success or failure, mission performance time, function times, task times, cognitive workload, and task failures. In addition IMPRINT output can include the effects of environmental variables such as heat, cold, and clothing. The predicted mission performance highlights issues that should be the focus of research studies, usability testing, and field tests as the system design matures. In turn, the completed models and predicted results are verified by 
SSME performance and feedback during usability studies and field tests during later stages of the acquisition cycle. Any required model modifications are made by the analysts and the revised models become the baseline for analyses of modifications to the system design for the next iteration of the acquisition cycle.

\subsection{Applying IMPRINT to Measure the UX}

Currently, MANPRINT practitioners are using IMPRINT to compare the human machine interface (HMI) of the manpack radio (AN/PRC-155), to a notional HMI for the radio. In this example, an analyst used the task-by-task instructions provided in the radio's operator manual to an operational vignette provide by SMEs to build an IMPRINT model that reflects how Soldiers are using the fielded radio during missions. Next, the analysts walked-through the model with SMEs and incorporated the operators' feedback within a revised IMPRINT model. To compare an alternative design, the analyst is building an IMPRINT model representing a notional radio concept in which the radio operator is guided through the radio operation by an operational aid or "wizard". Once again, SSMEs will provide feedback on the model and it will be revised if necessary. One performance measure that will be included within each model as output is the number of times that a radio operator requires the assistance of a maintainer to obtain help. Another is operator response time to critical messages transmitted or received. Mission performance measures will include quantitative measures such as wait time for the radio maintainer to respond, costs for radio maintainers, additional costs for training to task proficiency, mission failures due to lack of required message response time, and percentage of time in cognitive overload. Results are being provided to acquisition leadership to demonstrate the trade-offs between design for usability and its impact on training and performance. Because Soldiers were part of the model development process the results reflect user feedback and support UX design concepts. Because the results are quantified, leadership is likely to accept the trade-off data and this, in turn, should support selection of the optimum design.

\subsection{Applying Human Figure Modeling to Predict the UX}

One of the U.S. Army's programs which is primed to seek out more efficient ways of executing program acquisition while also integrating the UX is the Enhanced Medium Altitude Reconnaissance and Surveillance System (EMARSS). The EMARSS consists of a commercial derivation aircraft equipped with a variety of intelligence collection systems. It has leveraged 3-Dimensional (3D) human modeling to refine system design and examine design tradeoffs to increase the functionality of the workstation and the usability of its UIs. In doing so, the program was able to identify a serious safety concern prior to system design - the likelihood for a torso strike should the aircraft decelerate quickly - see Figure 1(a). These 3D models also facilitated the identification of physical dimension concerns that could potentially result in serious ailments after prolonged use due to the wrist angle for the operators (i.e., carpel tunnel syndrome) - see Figure 1(b). As a result of the 3D modeling efforts, the program was 
able to investigate and integrate an alternative seat into the aircraft which significantly mitigated these safety and physical dimension concerns.

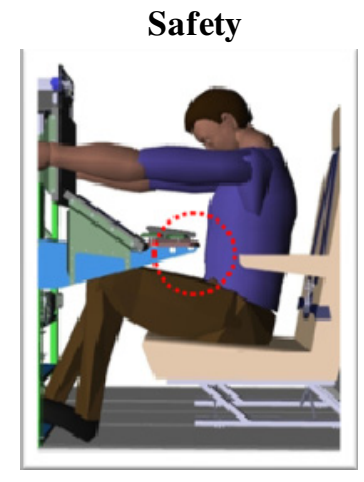

(a)
Physical Dimension

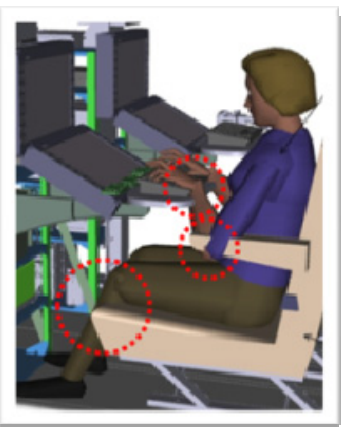

(b)

Fig. 1. EMARSS 3D modeling to identify UX issues early in the design process

\section{The U.S. Army Agile Process}

\subsection{Early UX Assessment during the NIE}

The NIE is a major test and evaluation event in which C4ISR equipment is assembled and configured to form the U.S. Army's tactical internet. There are many components that are in varying states of maturity with varying acquisition strategies. Some components are in the traditional acquisition process, others are a combination of commercial or modified commercial equipment, and many components are born from the Agile acquisition process. During NIE, a brigade combat team uses the equipment to conduct war games as part of their training while testing new capabilities. The team exercises its mission planning functions, intelligence gathering, and movement and maneuvers over a vast area in the desert with an opposing force that does the same.

In May and June 2011, the U.S. Army executed the first of six NIEs designed to test the effectiveness of networked technologies in an operational environment. The focus was on technical integration, and the intent was to determine how well these systems met the networking requirements of our Soldiers executing tactical missions. However, by the second test, NIE 12.2, system design attributes such as ease-of-use, accessibility, and trainability were recognized among core integration evaluation criteria. These criteria also became integral in the candidate assessment and source selection processes for future NIEs as part of the U.S. Army Agile Process (A2P).

The U.S. Army recognized that complex system designs increase training time for Soldiers to become proficient operators and maintainers; increase reliance on engineers/field service representatives (FSRs) to troubleshoot, fix, and repair systems in field; and degrade the collective unit's overall ability to complete tactical missions in a timely, safe, and efficient manner. According to the Government Accountability 
Office, after reviewing five NIEs the U.S. Army found it needed the ability to “...automatically reconfigure radios and other systems remotely ... these systems require intense pre-configuration and manual interface." [9, p. 16]. Additionally, the Director, Operational Test and Evaluation concluded in their review of NIEs in FY12 that the:

"Network component, both mission command systems and elements of the transport layer, are excessively complex to use. The current capability...is diminished due to pervasive task complexity. For example, what should be relatively simple tasks of starting up and shutting down systems requires complex series of actions by the operator" [10, p. 8].

Additional findings from the review noted that planning and loading tactical radios was cumbersome and time consuming processes; and that there is a distinct correlation between the dependency on FSRs and excessive complexity of using network systems.

In late FY13, in response to NIE findings and equipped with specific MANPRINT lessons learned from MANPRINT risk mitigation efforts on select U.S. Army programs, our organization partnered with the System of Systems Integration and Evaluation Directorate, of the Assistant Secretary of the Army for Acquisitions, Logistics, and Technology to ask the "right" questions of system developers, in the A2P. It was evident that U.S. Army officials and our Soldiers needed trained advocates, involved in initial system assessment, selection and evaluation to identify, discuss, and eliminate, where necessary, proposed system designs that did not fully consider how Soldiers would operate or even integrate the system into a network mission. We ensured that system providers supplied evidence that their systems were designed with the Soldier and their missions in mind. Thus, a complete answer was based on the presence of data or reports as evidence that the system: (1) underwent a Soldier-centric system design process, and (2) engineers were able to identify and describe Soldier critical tasks. Critical tasks were those that would result in unintended operational downtime or mission failure.

The A2P - MANPRINT Risk Readiness methodology and report engages the A2P through the Product Supportability Integrated Product/Process Team (PS IPT). The PS IPT, also referred to as Factor IV (Burdens), assesses systems under evaluation along lines of sustainment supportability, associated costs, and training requirements when compared to similar existing U.S. Army systems. These assessments are based on hardware and software support logistics, safety, survivability, reliability, maintainability, and MANPRINT aspects. In this forum, MANPRINT practitioners collaborate with logisticians, systems engineers, and reliability engineers to generate the initial white paper requirements by which industry candidates are evaluated; in generating specific requests for information for the technical interchange meeting and NIE Bull Pen (described in more detail below); and in coordinating and collaborating on systems under evaluation assessments generated during and post NIE execution.

One of the more significant achievements of the PS IPT has been the updated Product Support Questionnaire. This tool was fully updated and incorporated in the request for candidates to participate in NIE 14.2. More than $25 \%$ of the Product Support Questionnaire (or 13 of 50 questions) was either updated or new questions added to emphasize MANPRINT and system design characteristics. These questions 
include but are not limited to: "Does the system require 'round-the-clock' or sustained attention?" and "What types of evaluations of the system's Soldier-machine interfaces (physical and software) or usability have been performed?" The intent was to leverage the sources sought process as a forcing function for industry to more fully consider users' goals, intentions, and behaviors to improve Soldier-system interactions.

Another accomplishment for MANPRINT has been to have representation during the NIE Bull Pen. The NIE Bull Pen provides another touch-point in the A2P in which systems selected to participate in the NIE receive additional screening to understand their sustainment impact to the U.S. Army. This includes an assessment to understand burdens Soldiers may experience in system set-up, operations, transport, maintenance, and tear-down. Soldier burdens include identifying special tools and training necessary for troubleshooting and maintenance; understanding the time and the number of steps required to start-up or shut-down systems; and a discussion about the manpower and personnel skills required to successfully operate the system with minimal disruption to operational availability. This also includes determining the extent to which a system supports the information needs of its users to minimize operational downtime due to a loss of network, power, or incorrect system setup resulting in degraded or lost communication capabilities. This is discussed in greater detail pertaining to the evolution and operational testing of tactical radios in the next section. Findings from the Bull Pen inform evaluation and data collection priorities to ensure the UX is explicitly assessed as part of the A2P.

Fully integrating MANPRINT within the A2P through the PS IPT and NIE Bull Pen have increased the opportunities available to MANPRINT practitioners to emphasize the UX and to apply lessons learned from the field to influence the initial assessment, selection, and ongoing evaluation of future C4I systems.

\subsection{Assessing the UX During NIE Testing}

Over the last 25+ years, C4ISR technology has advanced exponentially. Tactical radios, once used primarily for voice communication and limited data transmission such as position reports, have been replaced by smaller and more capable devices that transmit and receive voice and enormous amounts of data traffic across the tactical internet which did not exist 20 years ago. Before 2000, tactical radios were typically large, heavy and had limited modes of operation. Radio control consisted of various knobs, switches, buttons (i.e., hard controls) with a small display used for system feedback. This old technology has been replaced by high powered computers that have operating systems, universal transceivers and store the software logic, algorithms, and operational data to emulate the capabilities of all the old radios that it replaces as well as new communication capabilities that tie into the tactical internet.

New C4ISR technology has allowed information to reach the battlefield's leading edge - the dismounted Soldier. The new generation radios serves as the conduit to the tactical internet, the US military's information highway. This presents a big UI design challenge. End user equipment operation for communicating voice and data across the battlefield must be simple, intuitive, require minimal attention demands, and training time. The equipment must not require any specialized skill more than 
that required to fight the fight, whether the Soldier is executing a mission on foot, riding in ground vehicle, or supporting ground troops while flying a helicopter.

To further add to the UI design challenges, C4ISR equipments must meet hundreds of performance requirements. Each performance requirement translates to one or more system design requirements, each which can potentially be decomposed into several derived design requirements. Considering system performance requirements, size, weight, power, environmental requirements, information assurance compliance requirements, and the general purpose user requirement, compromises must be made because many of the requirements do not co-exist very well. It is imperative that a qualified MANPRINT professional with UI design experience participates early in the system engineering process, understands the design challenges that the other engineering disciplines face, and is capable of identifying and communicating MANPRINT issues. Otherwise, there is a high degree of likelihood that the initial system design will have usability problems.

New generation multi-mode radios have been the centerpiece during NIE test events. While the radios are capable of interoperating with legacy radios, the new "digital radio" voice and data communication capability uses an internet protocol address to maintain connectivity with all the radios in a group. Large groups of users can split into smaller groups and merge back into a large group and maintain communications to support missions of the dismounted Soldier below the platoon level. Range extension radios strategically positioned on ground and aerial platforms can connect groups that may have split or connect two independent groups. Also, it is possible to monitor five groups of radio traffic at once whereby the groups monitored may only receive traffic from their own group. The concept of operation is quite different than what Soldiers have experienced using legacy equipment.

During system development, we support UI-centered design events, heuristic evaluations, and iterative usability testing as the design matures. Approximately half the systems participating at NIE have had some level of our support during development. Unlike UI test and evaluation activities that occur during system development, NIE evaluation can yield findings that could never have been made in a controlled test environment. This is because the equipment is being used in a manner and context truly representative of war fighting and the evaluator is exposed to the Soldier's experience to some degree (www.youtube. com/watch?v =Ovj4hFxko7c ). Our evaluators observe system training and communication exercises that test the system.

When the games begin, we travel to the test units during breaks in the mission during resupply and other logistics-related activities and interview users. These times are typically very early in the morning and very late in the evening. Soldiers are asked questions such as whether or not the equipment was used, was it needed, how well did it work, was it easy to use, and what improvements are recommended. When possible, Soldiers demonstrate problems that they experienced with the equipment. As the mission continues and the troops drive further into the desert, the evaluators coordinate with the test officer and visit the units while they are receiving logistics support. Missions occur over the course of three weeks and over time, users and evaluators get to know each other. Soldiers tend to become more comfortable with the evaluator and speak more freely with them allowing the evaluator more access to the user's world. 
Not only does the evaluator collect data on the equipment under evaluation, but they learn how the mission went the night before, the problems that were encountered when a vehicle made the wrong turn, an injury sustained during an accident, dynamics within the fighting unit, and the extreme cognitive and physical workload that users endure. If new to the U.S. Army culture, participating in the NIE is a great opportunity to learn how the U.S. Army does business. If one has served in the past, it is good opportunity to learn how it does business today. This insight into the user's experience is a great reminder that the equipment is merely a tool to assist Soldiers in executing their mission, in the case of tactical radios; their mission is not focused on operating radios.

Insight to the UX via NIE participation has allowed us to identify usability problems that are difficult to detect during heuristic reviews and usability evaluations. It is especially advantageous when we have had the opportunity to participate in UI development activities because this information enables us to give special attention to known UI weaknesses as NIE evaluators. Generally speaking, one of the most common usability problems that Soldiers experienced with the new generation of tactical radios was their inability to determine the radio's state of readiness following prolonged periods of non use. Soldiers needed to quickly assess whether the radio was set to communicate and make any changes if it was not. Soldiers had difficulty remembering how to accomplish this during times of stress and high workload. To add to the challenge, there are many processes that occur with networking radios that are transparent to the user. We documented the findings along with recommended solutions and passed them on to project management.

To mitigate communications issues that we observed at NIE, we entered into a partnership with the U.S. Army Training and Doctrine Command to study the relationship between usability and trainability. Refer to Section 2.1 for a discussion of this effort.

\subsection{Refining Training Based on UX Feedback}

During NIE, MANPRINT practitioners observe training and collect data via surveys and interviews from participating Soldiers. The data enables to understand the Training UX and revise programs of instruction to better meet the needs of Soldiers.

Initially, the Maintainer course was conducted over two days. Maintainers are the Soldiers who install, maintain, and troubleshoot signal support equipment and terminal devices. The first course was conducted with seven Soldiers and operator tasks were covered on the first day and troubleshooting the radio was covered on the second day. Troubleshooting encompassed using both the radio and a subset of the vehicle mounts to which it could be mounted to (e.g., tanks and other tactical ground vehicles) on tabletop trainers. All components associated with a radio's vehicle installation were provided with the exception of the actual vehicle. At the conclusion of the training, a group interview was conducted to obtain feedback. All Soldiers wanted the training to be increased to three days and suggested changes to the course structure. 
The Maintainers commented that the tabletop trainers worked well, but not every Maintainer was able to have hands on time with all three systems provided during the course. The Maintainers wanted everyone to have the opportunity to put each tabletop system together and break it down. This would allow them to ask questions and receive assistance from the training instructors if needed.

NIE provides the opportunity to follow up with the Soldiers during the test event. Three maintainers were interviewed during NIE to assess training coverage sufficiency for the problems that they encountered in the field and several additional coverage gaps were identified.

The Maintainer course was increased to three days and the tabletop trainers continue to be used. The focus has shifted to providing sufficient hands on time for the various vehicle installations and verifying that the training is providing the necessary information for maintainers to troubleshoot the radios and get them operational again.

\section{References}

1. Skelton, I.: MANPRINT for the US Army. Congressional Record-House, H8269-71 (1997)

2. Pew, R.W., Mavor, A.S. (eds.): Human-System Integration in the System Development Process: A New Look. National Academy Press, Washington, DC (2007)

3. Savage-Knepshield, P.A.: Soldier-Centered Design and Evaluation Techniques. In: Savage-Knepshield, P., Martin, J., Lockett III, J., Allender, L. (eds.) Designing Soldier Systems: Current Issues in Human Factors, pp. 275-307. Ashgate, Farnham (2012)

4. Army News Service: Army Demonstrates New Agile Acquisition Process to Industry Partners. DefenceTalk (2011), http://www.defencetalk.com/army-demonstratesnew-agile-acquisition-process-to-industry-partners-37071

5. Mitchell, D.K., Samms, C.L., Henthorn, T.J., Wojciechowski, J.Q.: Trade Study: A TwoVersus Three-Soldier Crew for the Mounted Combat System (MCS) and Other Future Combat System Platforms (ARL-TR-3026). Army Research Laboratory, Aberdeen Proving Ground (2003)

6. Mitchell, D.K., Brennan, G.: Soldier Workload Analysis of Infantry Vehicles With Alternative System Designs (ARL-TR-6375). Army Research Laboratory, Aberdeen Proving Ground (2013)

7. Mitchell, D.K., Brennan, G., Lobo, B.: Workload Analyses of Reconnaissance Vehicle Commander: With and Without Robotic Asset Responsibilities (ARL-TR-6607). U.S. Army Research Laboratory, Aberdeen Proving Ground (2013)

8. Mitchell, D.K.: Workload Analysis of the Crew of the Bradley Infantry Fighting Vehicle: Baseline IMPRINT Model (ARL-TR-6083), U.S. Army Research Laboratory, Aberdeen Proving Ground (2012)

9. U.S. Government Accountability Office: Army Networks: Opportunities Exist to Better Utilize Results from Network Integration Evaluations: Report to the SubCommittee on Tactical Air and Land Forces, Committee on Armed Services, House of Representatives (GAO Publication No. GAO-13-711) (2013), http://www.gao.gov/products / GAO-13-711

10. The Office of the Director, Operational Test and Evaluation: DOT\&E FY2013 Annual Report (2013), http: / /www. dote. osd.mil / pub/reports / FY2 013 